\title{
Fine Details Obtained by 3D Printing and Using Polymers
}

\author{
LAURENTIU SLATINEANU ${ }^{1}$, OANA DODUN ${ }^{1 *}$, GHEORGHE NAGIT ${ }^{1}$, MARGARETA COTEATA ${ }^{1}$, GHEORGHE BOSOANCA ${ }^{1}$, \\ IRINA BESLIU² \\ ${ }^{1}$ Gheorghe Asachi Technical University of lasi, Department of Machine Manufacturing Technology, 39A D. Mangeron, Blvd., \\ 700050 lasi, Romania \\ ${ }^{2}$ Stefan cel Mare University of Suceava, Department of Mechanics and Technology, 13 Universitatii Str., 720229 Suceava, Romania
}

\begin{abstract}
The three-dimensional printing is a manufacturing method involving the addition of materials by using certain principles valid in printing techniques. There are various techniques of a three-dimensional printing method and the most of them could be applied inclusively to generate objects of polymers. The objective of the research presented in this paper was to analyze the capabilities of 3D printing process or equipment of generating fine details and to identify a way of evaluating these capabilities when using polyester PLA as filament material. The systemic analysis of the printing techniques which use a fused polymer filament deposition showed that there are some groups of factors able to affect the obtaining of fine details. An experimental research was designed in order to highlight the influence exerted by the diameter of the nozzle orifice and by the values of sharp angles of isosceles triangles on the heights of these triangles, thus obtaining an image concerning the possibilities of generating sharp edges by three-dimensional printing. To evaluate the capacity of the 3D printing process of obtaining thin walls, a spiral including linear segments with a decreasing thickness from $1 \mathrm{~mm}$ was also achieved on the test piece. By mathematical processing of the experimental results using a specialized software, empirical mathematical models were determined to evaluate the intensity of influences exerted by the two process input factors on the heights corresponding to isosceles triangles characterized by sharp angles.
\end{abstract}

Keywords: 3D printing, polymer, fine details, influence factors, sharp edges, empirical models

In the last decades, the problem of rapid manufacturing of prototypes was intensively investigated. In this way, a new research field was gradually defined and afterward adequate applications were identified and optimized; initially, the researches covered by this investigation direction were included in the group of methods and techniques corresponding to the so-called rapid prototyping. The group was including various rapid manufacturing methods, like fused deposition modeling, stereolithography, selective laser sintering etc.

The rapid prototyping methods were allowing operative obtaining of simpler or more complex parts, initially made only of a restraint group of materials. One of the techniques developed in the last decades and which found various applications was the three-dimensional (3D) printing.

Essentially, the 3D printing process is used to create three-dimensional objects by means of a software that firstly ensures adequate relative movements between a source able to gradually generate the desired object by adding new layers of material and the platform on which the desired part is generated. This was the reason for which the researchers developed a new investigation direction corresponding to the additive manufacturing processes.

The first equipment in this field was designed and achieved by the Japanese researcher Hideo Kodama in the Nagoya Municipal Research Institute [1]. Kodama was the author of some patents proposing additive manufacturing methods by which three-dimensional plastic models were created using an ultraviolet exposure in the case of photo-hardening thermoset polymers. To control the area affected by the process, he used a mask pattern or a scanning fiber transmitter.

Actually, the 3D printing process has many and various applications, allowing obtaining of foods, metallic or plastic complex parts, cars components, spare parts, patientmatched implants, ornamental objects etc. The better knowing of the 3D printing process characteristics led to the development of a large equipment set able to be used for obtaining more and more complex parts or just assemblies of parts. In some cases, special requests were formulated for generating fine details by 3D printing. The researchers tried to know which could be the equipment capabilities of manufacturing thin walls, low values for corner radius, sharp angles of intersection between two flat surfaces etc.

In a paper concerning the principles and method for development of fused deposition modeling using minerals, Kochesfahani considered that the process of obtaining fine details could be affected by the temperature and viscosity of the melt. He proposed an optimized model of the fused deposition process, aiming to enhance the printing performance and print quality [2] .

Ramya and Vanapalli took into consideration various 3D printing methods, among these being analyzed the stereolithography, fused deposition modeling, laminated object manufacturing, selective laser sintering, direct metal laser sintering, ink jet printing and polyjet printing [2]. They appreciated that fine details could be obtained in the case of using poly-jet to produce toys. They noticed that exceptionally fine details and an outstanding surface finishing could be ensured by using a 3D printing process for producing dental parts [3].

Xiao et al. approached the problem of using color 3D printing technology to obtain facial soft tissue prostheses [4]. They observed that the 3D mesh generated by means of a 3D camera system could not consider fine details including pores and wrinkles. Another their remark refers to adding very fine details using finer pow der.

Olasek and Wiklak appreciated that the 3D printing process ensures obtaining complex model shape when it is applied to create objects purposed for aerodynamic testing [4]. They considered that the use of UV curable 
acrylic plastic in multi jet modeling 3D printing technology allows obtaining of very fine details of thin walls.

The objective of the research presented in this paper was to analyze the capabilities of 3D printing process or equipment of generating fine details and to identify a way of evaluating these capabilities.

\section{D Printing process}

Nowadays, two distinct essential principles solutions are used in order to develop 3D printing processes and they could be materialized by using the various distinct equipment.

A group of such 3D printing equipment uses a filament of material from which the desired part will be obtained. The filament is sent to a moving heating head from which the material of the filament is gradually deposited initially on a moving platform and subsequently, the layer by layer deposition contributes to the generation of the desired part (fig. 1). Both the platform on which gradually the part is generated, the heating head and the bar on which the heating head is placed move along established trajectories, elaborated by using an adequate software.

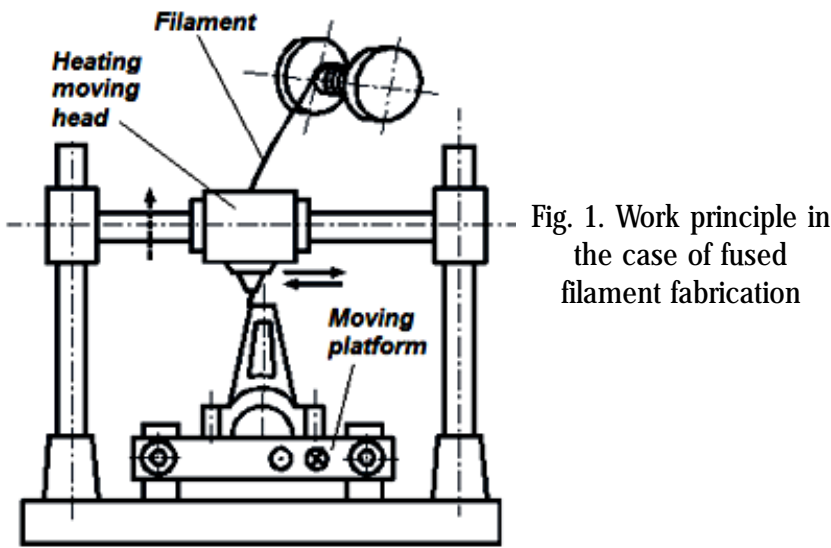

On the other hand, there are 3D printing techniques based on a process of liquid photopolymer polymerization. The polymerization develops due to the action of a light emitting source which directs a beam radiation of known geometrical and physical characteristics to a platform, through a transparent bottom of the recipient containing the liquid polymer (fig. 2). The platform achieved a vertical lifting movement so that an adding process of layer by

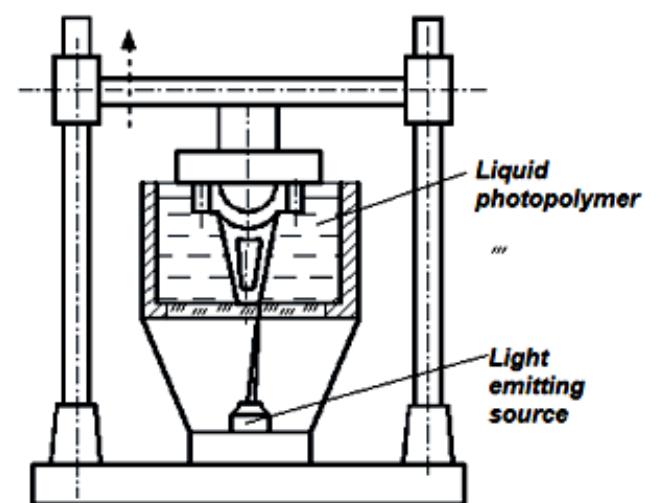

Fig. 2. Work principle in the case of stereolithography based on the use of a light-emitting source and a tank filled with a liquid photo-polymer resin

layer determines gradually the generation of the desired part.

A systemic analysis of the process of obtaining fine details by 3D printing and using polymers as filament materials highlighted the following groups of process input factors:

- Chemical composition of the filament material;

- Physical properties of the filament material;

- Geometrical characteristics of the nozzle orifice (shape of orifice cross and longitudinal section, orifice surface roughness etc.);

- Position of the nozzle to the platform;

- Temperature of the filament material when leaving the nozzle;

- The accuracy of relative movements between nozzle and platform etc.

As process output parameters valid in the case of the process of obtaining fine details by $3 \mathrm{D}$ printing and using polymers as filament materials, one could consider:

- Dimensional, positioning and shape accuracy of the obtained surfaces;

- Roughness of the generated surfaces;

- Corner radius of edges rounding;

- Values of sharp angles betw een two flatsurfaces which intersect etc.

A graphical representation elaborated in order to illustrate the results of the systemic analysis applied to the process of 3D printing when using polymers as filament materials is presented in figure 3 .

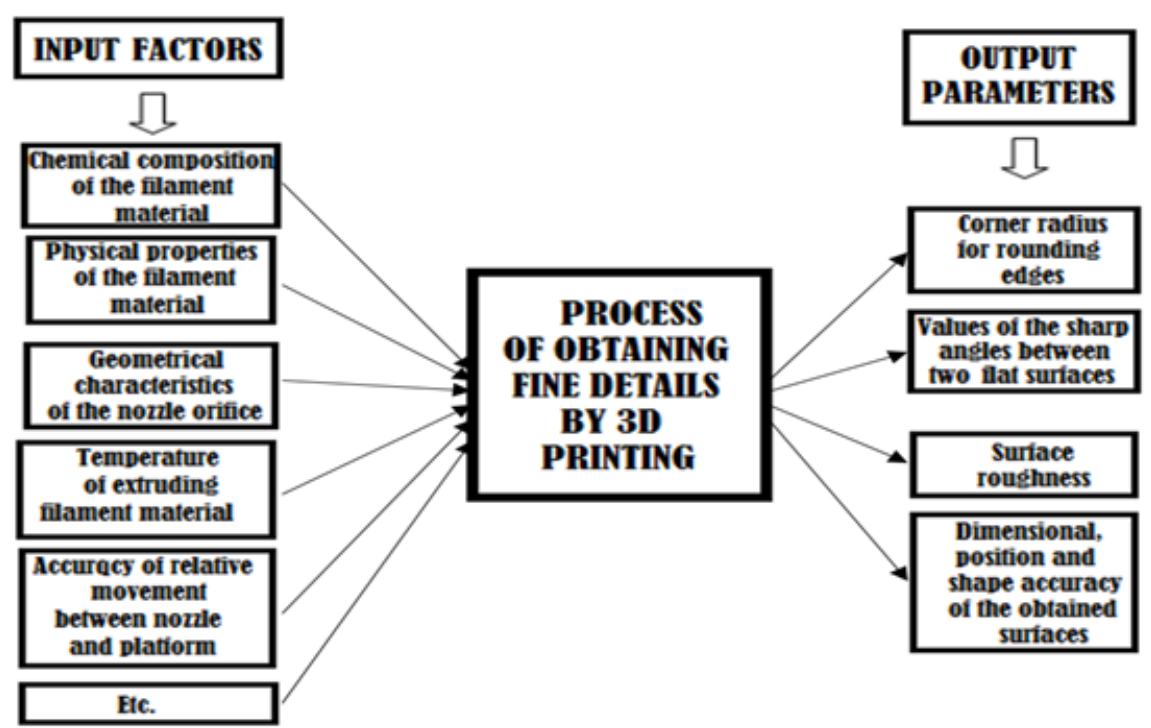

Fig. 3. Results of systemic analysis applied to the process of obtaining fine details by 3D printing when using polymers as filament materials 


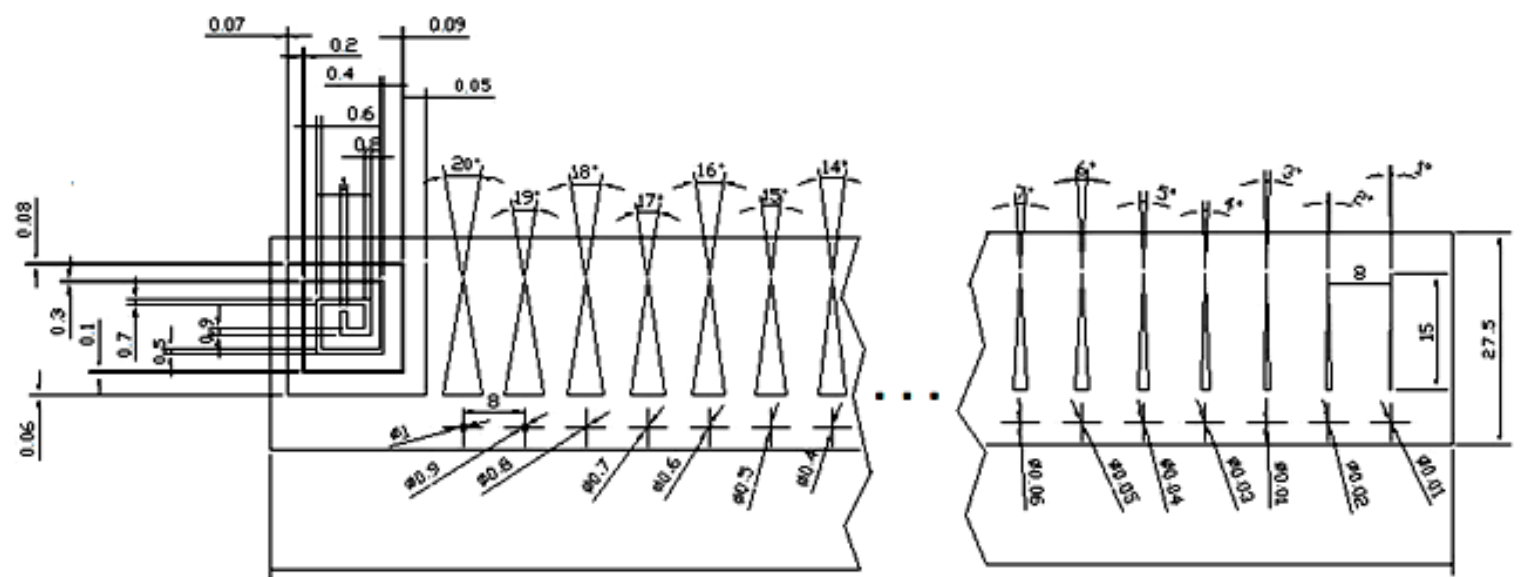

Fig. 4. Essential dimensions of the test sample designed in order to investigate the capacity of obtaining fine details by 3D printing

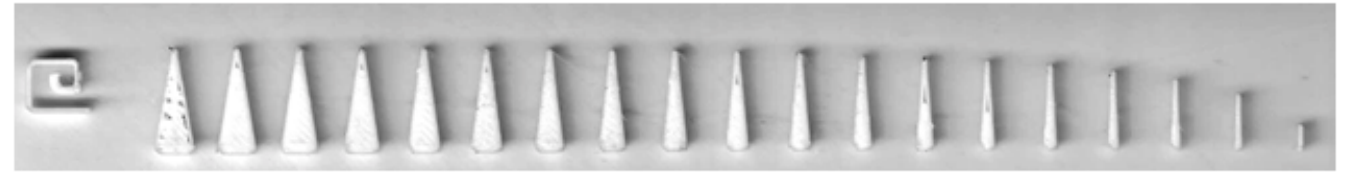

Fig. 5. Image of one of test pieces obtained by using the designed part from figure 4

\section{Experimental part}

The objective of the research presented in this paper was to obtain an image concerning the possibility of obtaining fine details by 3D printing and using fused filament deposition method.

A usual 3D printing equipment (type SmartRap+) was used within experimental research. A filament made of polyester PLA (frequently called polylactic acid, although this name does not correspond to the standard nomenclature promoted by the International Union of Pure and Applied Chemistry) was fused and passed through nozzles having holes of two diameters $(0.1$ and $0.2 \mathrm{~mm})$. The polylactic acid PLA is considered as a biodegradable and bioactive thermoplastic aliphatic polyester and it can be obtained especially from renewable resources.

The 3D filament had a diameter of $1.75 \mathrm{~mm}$ and was produced by Devil Design (Poland). The filament material had a density of $1.210-1.430 \mathrm{~g} / \mathrm{cm}^{3}$ and a melting point of $150-160^{\circ} \mathrm{C}$. The melting temperature for the $3 \mathrm{D}$ printing process was about $205^{\circ} \mathrm{C}$, while the temperature of the support was of about $70^{\circ} \mathrm{C}$. A layer thickness of $0.1 \mathrm{~mm}$ was obtained. The programmed printing speed was 30 $\mathrm{mm} / \mathrm{s}$.

In order to investigate the equipment capacity of achieving fine details, the problem of fabricating a set of isosceles triangles with decreasing values of the sharp angles was stated (fig. 4).

There was yet the intention to evaluate the capacity of $3 D$ printing process of obtaining thin walls; with this aim in view, a spiral including linear segments with a decreasing width from $1 \mathrm{~mm}$ with a step of $0.1 \mathrm{~mm}$ was proposed to be achieved (the first segment had a width of $1 \mathrm{~mm}$, the second - of $0.9 \mathrm{~mm}$, the third - of $0.8 \mathrm{~mm}$ and so on). This spiral could be observed in the left zone of figures 4 and 5 ; one could see that the lastsegment achieved by 3D printing has a width of $0.5 \mathrm{~mm}$ both in the case of orifice nozzle diameter $d=0.1 \mathrm{~mm}$ and $d=0.2 \mathrm{~mm}$.

An image of one of the test pieces obtained in the abovementioned conditions is presented in figure 5 .

\section{Results and discussions}

The heights of the triangles found on the achieved parts were measured by means of an optical microscope and the obtained values were included in table 1 , for two situations, characterized by using two nozzles with distinct orifice diameters.
The experimental results were processed by means of a software based on the method of least squares [6-7], in order to establish empirical mathematical models able to highlight the influence exerted by the value of the sharp angle $\alpha$ of surfaces intersections and by the diameter $d$ of the nozzle orifice on the height $h$ of the triangles obtained by 3D printing. The software was elaborated in the Department of Machine Manufacturing Department of the Gheorghe Asachi Technical University of la'i and it offered the possibility to select an adequate model for the experimental results, among some distinct mathematical models. As a criterion for assessing the adequacy of a certain mathematical model to experimental results, the value of the Gauss's criterion was used.

The value of this criterion is determined as a sum of least squares corresponding to differences between the measured values and the values allocated on the base of the proposed mathematical model, for the same experimental points. The lower is the value of the Gauss's criterion, the more adequate the selected mathematical model is. In the case of the experimental results included in Table 1, among the available mathematical models, an adequate mathematical model could be considered the hyperbolic model, expressed by the relation:

$$
h=14.493+\frac{0.02811}{d}-\frac{23.885}{\alpha},
$$

for which the value of the Gauss's criterion is $S_{G}=0.2636711$.

Taking into consideration that in the case of a monotone variation of the output parameter (height $h$ of the isosceles triangle) when the values of the input factors change and appreciating that a pow er type function could offer more directinformation concerning the intensity of the influence exerted by the process input factors on the output parameter, a power type empirical function was also determined:

$$
h=5.532 d^{-0.0229} \alpha^{0.305},
$$

in this case the value of the Gauss's criterion being $S_{G}=0.6146371$.

A graphical representation elaborated on the base of the empirical mathematical relation (2) is presented in figure 6 . 
Table 1

MEASURED HEIGHTS OF ISOSCELES TRIANGLES HAVING DECREASING VALUES OF THE PEAKS ANGLES

\begin{tabular}{|c|c|c|c|c|c|c|c|c|c|c|c|c|c|c|c|c|c|c|}
\hline & \multicolumn{18}{|c|}{ Heights of isosceles triangles, $\mathrm{mm}$} \\
\hline $\begin{array}{c}\text { Colum } \\
\text { no. } 1\end{array}$ & 2 & 3 & 4 & 5 & 6 & 7 & 8 & 9 & 10 & 11 & 12 & 13 & 14 & 15 & 16 & 17 & 18 & 19 \\
\hline $\begin{array}{c}\text { Sharp } \\
\text { angle } \alpha \\
\text { of isos- } \\
\text { celes } \\
\text { triangle }\end{array}$ & 20 & 19 & 18 & 17 & 16 & 15 & 14 & 13 & 12 & 11 & 10 & 9 & 8 & 7 & 6 & 5 & 4 & 3 \\
\hline $\begin{array}{c}\text { Orifice } \\
\text { diameter } \\
d=0.1 \\
\mathrm{~mm}\end{array}$ & 13.01 & 13.65 & 13.66 & 13.56 & 13.03 & 12.98 & 13.21 & 13.00 & 12.84 & 12.59 & 12.55 & 11.98 & 11.67 & 11.34 & 10.85 & 10.02 & 8.97 & 6.92 \\
\hline \begin{tabular}{|c|} 
Orifice \\
diameter \\
$d=0.2$ \\
$\mathrm{~mm}$ \\
\end{tabular} & 13.70 & 13.87 & 13.75 & 13.54 & 12.98 & 13.09 & 13.06 & 13.02 & 12.74 & 12.75 & 10.30 & 10.29 & 12.03 & 11.67 & 11.09 & 10.18 & 9.11 & 6.13 \\
\hline
\end{tabular}

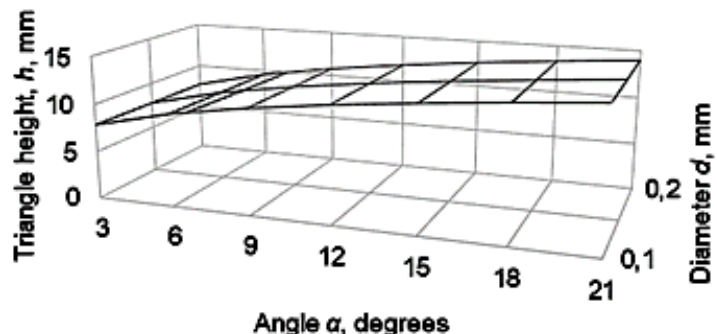

Fig. 6. Influence of the angle $\alpha$ and nozzle orifice diameter $d$ on the isosceles triangle height $h$, in accordance with the mathematical relation (2)

The analysis of the empirical mathematical relation (2) and of the graphical representation from figure 6 showed that, as expected, when the value of sharp angle of the isosceles triangle increases, the height of the achieved triangle increases, due to the $3 \mathrm{D}$ printing equipment capabilities; practically, if the value of the sharp angle is too low, there is not the possibility to materialize this zone of the triangle or just the triangle could not be achieved. In fact, the last tw 0 triangles (having $\alpha=2^{\circ}$ and $\alpha=10^{\circ}$ were not obtained on the test piece.

If the diameter of the nozzle hole is considered, one could notice that for its available interval of variation, the influence exerted on the height of the triangle is low enough, since the exponent attached to the factor $d$ in the relation (2) is very low.

The surface roughness of the surfaces obtained by 3D printing is also a parameter of technological and practical interest, but in this study, the surface roughness was not taken into consideration. As one can see in figur e 4, certain voids could be observed; they are the results of the 3D printing process characteristics. One could mention that due to these characteristics, it is difficult to obtain parts with a high level of compactness.

\section{Conclusions}

The 3D printing is one of the mostapplied manufacturing methods for obtaining parts by material addition. Among the characteristics of this additive manufacturing method, sometimes the capacity of achieving fine details could be a factor that must be considered. A systemic analysis showed that there are some groups of factors able to affect the capacity of the 3D printing process when using fused polymer filament to obtain fine details. An experimental research was designed and materialized to evaluate the capacity of the used 3D printing equipment and process to obtain thin walls and low values of the sharp angles resulted by the intersection of two flat surfaces. The experimental results were mathematically processed and empirical mathematical models were determined, aiming to highlight the intensity of the influence exerted by the diameter of the nozzle orifice and by the value of the sharp angle corresponding to an isosceles triangle on the height of a triangle generated on a test piece. One noticed that the value of the isosceles triangle top angle exerts a significant influence on the height of the obtained triangle, and, for the considered variation interval, the nozzle orifice diameter proved only a reduced influence. In the future, there is the intention to extend the theoretical and experimental researches about the possibilities of the 3D printing process and equipment of obtaining fine details when using polymer fused filament.

\section{References}

1.KODAMA H., Review of Scientific Instruments, 52, 1981, p. 1770;

2. KOCHESFAHANI S.H., SPE ANTEC ${ }^{\text {TM }}$, Indianapolis, 2016, p. 1598;

3. RAMYA A., VANAPALLI S., International J ournal of Mechanical Engineering and Technology (IJ MET), 7, 2016, p. 396;

4. XIAO K., WUERGER S., MOSTAFA F., SOHAIB A., YATES J .M., InTech, 2016, p. 89;

5. OLASEK K., WIKLAK P., J ournal of Physics: Conference Series, 530, 2014, 012009;

6. ROSCULET, R., T., FETECAU, C., Journal of Engineering Sciences and Innovation, Volume 3, Issue 1/2018, pp. 15-24;

7. CRETU G., Fundamentals of experimental research. Laboratory handbook (in Romanian), Technical University of lasi, Romania, 1992.

Manuscript received: 15.09 .2018 